\title{
Gorenstein flat precovers and Gorenstein injective preenvelopes in Grothendieck categories
}

\author{
Edgar Enochs, J.R. García Rozas, Luis Oyonarte and Blas Torrecillas
}

\begin{abstract}
Homology theory relative to classes of objects other than those of projective or injective objects in abelian categories has been widely studied in the last years, giving a special relevance to Gorenstein homological algebra.

We prove the existence of Gorenstein flat precovers in any locally finitely presented Grothendieck category in which the class of flat objects is closed under extensions, the existence of Gorenstein injective preenvelopes in any locally noetherian Grothendieck category in which the class of all Gorenstein injective objects is closed under direct products, and the existence of special Gorenstein injective preenvelopes in locally noetherian Grothendieck categories with a generator lying in the left orthogonal class to that of Gorenstein injective objects.
\end{abstract}

\section{Introduction}

The study of the existence of Gorenstein injective preenvelopes and Gorenstein flat precovers has been subject of much research in recent years. From the category of modules to the category of representation of quivers by modules or the category of sheaves of modules over a topological space, and so in categories such as that of graded modules, or that of complexes of modules or the one of discrete modules over a profinite group, the knowledge of the existence of these precovers or preenvelopes is of great interest in order to be able to develop relative homology.

There have been many results in this area proving the existence of these type of precovers/preenvelopes in some of the categories mentioned above when some

Key words and phrases: Gorenstein flat object, Gorenstein injective object, Grothendieck category, precover, preenvelope.

2010 Mathematics Subject Classification: 18G25. 
conditions are satisfied. But such an existence is of interest in every category where computing homology makes any sense, so in this paper we try to find the minimal conditions that a (Grothendieck) category must satisfy to guarantee that Gorenstein injective preenvelopes or Gorenstein flat precovers exist.

Of course this question has been studied already by several authors and some interesting results have been proved in this direction, so for instance Krause proved in [21, Theorem 3.7.4] that special Gorenstein injective preenvelopes always exist in locally noetherian Grothendieck categories whose derived category is a compactly generated triangulated category; Enochs et al. showed ([4, Theorem 2.25]) that in any Gorenstein category the pair $(\mathcal{L}, \mathcal{G I})(\mathcal{L}$ is the class of objects of finite projective dimension) is a complete hereditary cotorsion theory (so in particular every object of the category has a special Gorenstein injective preenvelope); Yang and Ding showed in [28, Proposition 4.8] that if the category is a locally noetherian Grothendieck category and has a generator of finite injective dimension, then the pair $(\mathcal{L}, \mathcal{G I})$ (where $\mathcal{L}$ is the class of all objects of injective dimension less than or equal to that of the generator) is a complete hereditary cotorsion theory (so again every object has a special Gorenstein injective preenvelope); or Gillespie proved ([16, Corollary 7.7]) that if the category is locally finite dimensionally type $F P_{\infty}$ and $\mathcal{G} \mathcal{A C}$ is the class of all Gorenstein $\mathcal{A C}$-injective objects, then the pair $\left({ }^{\perp} \mathcal{G} \mathcal{A C}, \mathcal{G} \mathcal{A C}\right)$ is a cotorsion theory cogenerated by a set and ${ }^{\perp} \mathcal{G} \mathcal{A C}$ contains a set of generators of finite projective dimension and of type $F P_{\infty}$ of the category. This means that every object has a special $\mathcal{G} \mathcal{A C}$-preenvelope. In the particular case in which the category is locally noetherian (with a set of generators of finite projective dimension), the class $\mathcal{G} \mathcal{A C}$ coincides with $\mathcal{G} \mathcal{I}$ so we have special Gorenstein injective preenvelopes.

In this paper we prove that in a locally noetherian Grothendieck category in which the class of all Gorenstein injective objects is closed under direct products, Gorenstein injective preenvelopes always exist. If, in addition, we want to get special Gorenstein injective preenvelopes, we will require the category to have a generator lying in the class ${ }^{\perp} \mathcal{G} \mathcal{I}$ (we will notice that if this condition holds then direct products of Gorenstein injectives are Gorenstein injective). As a consequence, we will show that the pair $\left({ }^{\perp} \mathcal{G I}, \mathcal{G I}\right)$ is a complete hereditary cotorsion theory. This will give a different approach to those of [21] and [4], will properly extend Yang and Ding's result (since such categories include all module categories over noetherian rings, while not every noetherian ring has finite injective dimension), and will also extend Gillespie's result (the finite projective dimensional generators of the locally noetherian category hold in ${ }^{\perp} \mathcal{G} \mathcal{I}$ by [16, Lemma 7.2]).

As for the Gorenstein flat precovers, the most general results proved so far are due to Yang and Liang, who showed the existence of Gorenstein flat precovers for 
any module over any ring ([29, Theorem A]), and Christensen, Estrada and Iacob ([2]) who did the same in the categories of quasi-coherent sheaves over any scheme. In this paper we prove the existence of Gorenstein flat precovers in any locally finitely presented Grothendieck category whose class of flat objects is closed under extensions (so we properly extend the results of both papers). Indeed, we give a wide notion of flat objects (and so of Gorenstein flat objects) that in particular covers the definition of what flat means in categories such as that of modules, that of quasicoherent sheaves over a scheme or that of representations of quivers by modules. In other words, we prove the existence, in any locally finitely presented Grothendieck category with an extra condition, of some type of precovers that include Gorenstein flat precovers.

The paper is organized as follows: Section 3 is devoted to prove a result that will be used along the paper to prove two of our main results. This result gives the necessary conditions to transfer the property of the existence of certain type of precovers or preenvelopes from the category of complexes over a given locally finitely presented Grothendieck category to the original category.

In Section 4 we shall prove the existence of Gorenstein flat precovers in any locally finitely presented Grothendieck category provided that the class of flat objects is closed under extensions. To prove such a result we shall make use of the category of complexes over the original Grothendieck category and the existence of a certain type of precovers in these categories. This enables us to avoid the computations with Tor existing in the literature.

Section 5 is devoted to prove that Gorenstein injective preenvelopes always exist in locally noetherian Grothendieck categories provided that the class of Gorenstein injective objects is closed under direct products, and in Section 6 we prove that, again in locally noetherian Grothendieck categories, if a generator is in the left orthogonal class to that of all Gorenstein injective objects, then the existence of special Gorenstein injective preenvelopes is guaranteed for any object of the category.

Finally, in Section 7 we apply the results proved throughout the paper to the categories of special interest mentioned above.

\section{Preliminaries}

All categories used in this paper will be, unless otherwise specified, locally finitely presented Grothendieck categories. Given any such category $\mathcal{A}$, the category of complexes on $\mathcal{A}$ will be denoted as $C(\mathcal{A})$, and complexes will always increase the degree, so a complex will then be of the form

$$
\ldots \longrightarrow X^{n-1} \stackrel{\delta^{n-1}}{\longrightarrow} X^{n} \stackrel{\delta^{n}}{\longrightarrow} X^{n+1} \longrightarrow \ldots
$$


Given an object $M$ of $\mathcal{A}$, the $n$-disk complex over $M, D^{n}(M)$, will be the complex

$$
\ldots \longrightarrow 0 \longrightarrow M \stackrel{1_{M}}{\longrightarrow} M \longrightarrow 0 \longrightarrow \ldots
$$

where all entries are zero but the $n$-th and $(n+1)$-th entries. Similarly the $n$-sphere, $S^{n}(M)$, will be

$$
\ldots \longrightarrow 0 \longrightarrow M \longrightarrow 0 \longrightarrow \ldots
$$

with $M$ in the $n$-th entry.

Recall that given a complex $X$, the symbol $Z^{n}(X)$ is used to denote the kernel of the differential $\delta^{n}: X^{n} \rightarrow X^{n+1}$ and it's called the $n$-cycles object of $X$. Similarly $B^{n}(X)$ is used to denote the $(n-1)$-boundary of $X$. We use $Z(X)$ and $B(X)$ to denote the subcomplexes of $X$ whose components of degree $n$ are $Z^{n}(X)$ and $B^{n}(X)$ respectively, with the induced (zero) differentials.

Given a class of objects $\mathcal{F}$ of $\mathcal{A}$, a complex $X^{*}$ on $\mathcal{A}$ is said to be $\operatorname{Hom}(\mathcal{F},-)$ exact $\left(\operatorname{Hom}(-, \mathcal{F})\right.$-exact) if the complex $\operatorname{Hom}\left(F, X^{*}\right)\left(\operatorname{Hom}\left(X^{*}, F\right)\right)$ is exact for any object $F$ of $\mathcal{F}$. The class of all injective (projective) objects of $\mathcal{A}$ will be denoted as $\mathcal{I} n j(\mathcal{P} r o j)$.

Given a class of objects $\mathcal{F}$ in a category $\mathcal{C}$, its left orthogonal, ${ }^{\perp} \mathcal{F}$, is the class of all objects $C$ of $\mathcal{C}$ such that $\operatorname{Ext}_{\mathcal{C}}^{1}(C, F)=0$ for any object $F$ of $\mathcal{F}$. Similarly, the right orthogonal class of $\mathcal{F}, \mathcal{F}^{\perp}$, is that of all objects $C$ of $\mathcal{C}$ such that $\operatorname{Ext}_{\mathcal{C}}^{1}(F, C)=0$ for every object $F$ of $\mathcal{F}$.

An ordered pair of classes $(\mathcal{X}, \mathcal{Y})$ is said to be a cotorsion theory provided that $\mathcal{X}={ }^{\perp} \mathcal{Y}$ and $\mathcal{Y}=\mathcal{X}^{\perp}$. A cotorsion theory $(\mathcal{X}, \mathcal{Y})$ is said to be:

a) cogenerated by a set $S$ if $\mathcal{Y}=S^{\perp}$,

b) complete if for any object $O$ of the category there exist two exact sequences

$$
\begin{aligned}
& 0 \longrightarrow O \longrightarrow Y \longrightarrow X \longrightarrow 0 \\
& 0 \longrightarrow Y^{\prime} \longrightarrow X^{\prime} \longrightarrow O \longrightarrow 0
\end{aligned}
$$

with $X, X^{\prime} \in \mathcal{X}$ and $Y, Y^{\prime} \in \mathcal{Y}$.

c) hereditary if $\operatorname{Ext}^{i}(X, Y)=0 \forall X \in \mathcal{X}, \forall Y \in \mathcal{Y}, \forall i>0$. This is equivalent to $\mathcal{Y}$ being closed under cokernels of monomorphisms, and if the category has enough projectives then the cotorsion theory being hereditary is equivalent to $\mathcal{X}$ being closed under kernels of epimorphisms.

Recall that given a class of objects $\mathcal{F}$ in $\mathcal{C}$, an $\mathcal{F}$-precover (preenvelope) of an object $C$ of $\mathcal{C}$ is a morphism $\varphi: F \rightarrow C(\varphi: C \rightarrow F)$ with $F$ in the class $\mathcal{F}$, such that $\operatorname{Hom}_{\mathcal{C}}\left(F^{\prime}, \varphi\right)\left(\operatorname{Hom}_{\mathcal{C}}\left(\varphi, F^{\prime}\right)\right)$ is surjective for any object $F^{\prime}$ of $\mathcal{F}$. $\varphi$ is a special precover (preenvelope) if it is an epimorphism (monomorphism) an its kernel (cokernel) is an object of $\mathcal{F}^{\perp}\left({ }^{\perp} \mathcal{F}\right)$. $\varphi$ is an $\mathcal{F}$-cover $(\mathcal{F}$-envelope) if it is an $\mathcal{F}$-precover $(\mathcal{F}$ preenvelope) and any $f: F \rightarrow F$ with $\varphi f=\varphi(f \varphi=\varphi)$ is an automorphism. A class 
$\mathcal{F}$ is said to be precovering (preenveloping) if $\mathcal{F}$-precovers ( $\mathcal{F}$-preenvelopes) exist for any object of the category, and it is special precovering (special preenveloping) if every object has a special $\mathcal{F}$-precover (a special $\mathcal{F}$-preenvelope).

Throughout all this paper we will need the use of cardinalities of objects. These were studied in [4], where all basic properties that relate the cardinality of an object to that of one of its subobjects, epimorphic images and its injective envelope, were proved. For completeness we now list (without proofs) all the results of this type from [4] that we will need, plus some easy consequences of them that we will also use.

We will always work with locally finitely presented Grothendieck categories, so we will always have in hand a family of locally finitely presented generators. From now on we will let $\mathcal{A}$ and $\mathcal{B}$ denote two locally finitely presented Grothendieck categories (unless otherwise specified) and $H$ be the direct sum of a copy of each one of its finitely generated generators (either in the category $\mathcal{A}$ or $\mathcal{B}$, depending on where we will be working). With this generator in hand we can define what the cardinality of an object is.

Definition 2.1. [4, Section 2] The cardinality of any object $O$ is defined as the cardinality of the abelian group $\operatorname{Hom}_{\mathcal{A}}(H, O)$,

$$
|O|=\left|\operatorname{Hom}_{\mathcal{A}}(H, O)\right| \text {. }
$$

Proposition 2.2. ([4, Corollary 2.3]) Given any object $Y$ of $\mathcal{A}$, there is a cardinal number $\varkappa$ such that if $U \rightarrow Y$ is an epimorphism then there is a subobject $U^{\prime} \leq U$ such that $U^{\prime} \rightarrow Y$ is an epimorphism and that $\left|U^{\prime}\right| \leq \varkappa$.

Proposition 2.3. ([4, Corollary 2.4]) For every cardinal $\varkappa$ there is a set of representatives of objects $Y$ with $|Y| \leq \varkappa$.

Proposition 2.4. ([4, Lemma 2.5]) If $Z \leq Y$ is any subobject then $|Z| \leq|Y|$.

Proposition 2.5. ([4, Lemma 2.6]) Given a cardinal number $\varkappa$ there exists a cardinal number $\lambda$ such that if $|Y| \leq \varkappa$ and $Z \leq Y$ then $|Y| Z \mid \leq \lambda$.

Proposition 2.6. ([4, Lemma 2.7]) For any object $Y$ and any set $I$ we have $\left|Y^{(I)}\right| \leq\left|Y^{I}\right|=|Y|^{|I|}$.

Proposition 2.7. ([4, Lemma 2.9]) If $\gamma$ is an ordinal number and if $\left(\varkappa_{\alpha}\right)_{\alpha<\gamma}$ is a family of cardinal numbers, then there is a cardinal number $\lambda$ such that if $\left(Y_{\alpha}\right)_{\alpha<\gamma}$ is a family of objects with $Y_{\alpha} \leq Y_{\alpha^{\prime}}$ whenever $\alpha \leq \alpha^{\prime}<\gamma$, and such that $\left|Y_{\alpha}\right| \leq \varkappa_{\alpha}$ for each $\alpha<\gamma$, then $\left|\cup Y_{\alpha}\right| \leq \lambda$.

Proposition 2.8. ([4, Lemma 2.10]) Given a cardinal $\varkappa$ there is a cardinal $\lambda$ such that if $|Y| \leq \varkappa$ then $|E(Y)| \leq \lambda$ where $E(Y)$ is the injective envelope of $Y$. 
There is a natural property that holds in all of our categories (locally finitely presented Grothendieck categories) which we will make use of along the paper, but that needs to be checked out.

Proposition 2.9. Given any two cardinal numbers $\aleph$ and $\lambda$ with $|H| \leq \aleph$, there exists a cardinal number $\nu$ such that for any object $O$ and any family of subobjects $\left\{O_{i} \leq O ; i \in I\right\}$ with $|I| \leq \lambda$ and $\left|O_{i}\right| \leq \aleph \forall i$, the inequality $\left|\sum_{i \in I} O_{i}\right| \leq \nu$ holds.

Proof. We know there is an epimorphism $H^{\left(\left|\oplus_{I} O_{i}\right|\right)} \rightarrow \sum_{I} O_{i}$ (since we have two natural epimorphisms $H^{\left(\left|\oplus_{I} O_{i}\right|\right)} \rightarrow \oplus_{I} O_{i}$ and $\left.\oplus_{I} O_{i} \rightarrow \sum_{I} O_{i}\right)$. But applying twice Proposition 2.6 we get

$$
\left|H^{\left(\left|\oplus_{I} O_{i}\right|\right)}\right| \leq|H|^{\left|\oplus_{I} O_{i}\right|} \leq \aleph^{\left|\oplus_{I} O_{i}\right|} \leq \aleph \prod_{I}\left|O_{i}\right| \leq \aleph^{\aleph^{|I|}} \leq \aleph^{\aleph^{\lambda}} .
$$

Applying Proposition 2.5 we find $\nu$ with $\left|\sum_{I} O_{i}\right| \leq \nu$.

We now prove that the cardinality of any finitely generated object can be bounded by a single cardinal number

Proposition 2.10. There is a cardinal number $\lambda$ that upper bounds the cardinality of every finitely generated object.

Proof. Let $\varkappa$ be the cardinality of the generator, $|H|=\varkappa$. Then, for any finite set $I$ we have $\left|H^{(I)}\right| \leq \varkappa^{|I|}=\varkappa$ (provided that $\varkappa$ is infinite). Thus, by Proposition 2.5 we find a cardinal number $\lambda$ such that the image of any morphism from any $H^{(I)}$ ( $I$ finite) has cardinality $\leq \lambda$.

Now, Gorenstein injective and Gorenstein projective objects in categories have already appeared in the literature. We now recall these concepts.

Definition 2.11. An object $M$ of an abelian category $\mathcal{A}$ will be said to be Gorenstein injective (Gorenstein projective) provided that there exists an exact and $\operatorname{Hom}(\mathcal{I} n j,-)$-exact $(\operatorname{Hom}(-, \mathcal{P} r o j)$-exact) complex of injective (projective) objects

$$
\ldots \longrightarrow E^{-1} \longrightarrow E^{0} \longrightarrow E^{1} \longrightarrow \ldots
$$

such that $M=\operatorname{ker}\left(E^{0} \rightarrow E^{1}\right)$.

We close this section by defining locally noetherian (Grothendieck) categories.

Definition 2.12. An object $C$ of a Grothendieck category $\mathcal{A}$ is said to be noetherian if each one of its subobjects is finitely generated.

The category is $\mathcal{A}$ is locally noetherian if it has a family of noetherian generators.

It is well known that any locally noetherian category is locally finitely presented. The reader can easily prove this by referring to [26, Propositions 4.1 and 4.2 and paragraph before Proposition 4.1]. 


\section{A transfer property}

The following result was suggested by the referee in order to simplify and unify the arguments given in the original proofs of theorems 4.14 and 6.1 .

Proposition 3.1. Let $\mathcal{A}$ be an abelian category and let $\mathcal{E} \subseteq C(\mathcal{A})$ be any class consisting of exact complexes such that $\mathcal{E}=\mathcal{E}[1]$. Denote by $\mathcal{Z}(\mathcal{E})$ the class of all objects of $\mathcal{A}$ consisting of all zero-cycles of complexes in $\mathcal{E}$. The following statements hold:

a) If $\mathcal{E}$ is preenveloping (precovering) in $C(\mathcal{A})$ then $\mathcal{Z}(\mathcal{E})$-is preenveloping (precovering) in $\mathcal{A}$.

b) Suppose $\mathcal{Y}$ is a class of injective (projective) objects, let $\mathcal{T} \subseteq \mathcal{Y}^{\perp}\left(\mathcal{T} \subseteq{ }^{\perp} \mathcal{Y}\right.$ ) be any class of objects and let $\mathcal{E}$ be the class of exact complexes of objects in $\mathcal{Y}$ with cycles in $\mathcal{T}$. If $\mathcal{E}$ is special preenveloping (special precovering) in $C(\mathcal{A})$ then $\mathcal{Z}(\mathcal{E})$-is special preenveloping (special precovering) in $\mathcal{A}$.

c) Let $\mathcal{Y}$ and $\mathcal{T}$ be as in b), and $\mathcal{E}$ be the class of all exact complexes with components in $\mathcal{Y}$ and cycles in $\mathcal{T}$. If $\mathcal{E}$ is enveloping (covering) in $C(\mathcal{A})$ then $\mathcal{Z}(\mathcal{E})$-is enveloping (covering) in $\mathcal{A}$.

Proof. We will just prove the statements concerning preenvelopes. The precovers version is completely dual.

a) For any object $M$ of $\mathcal{A}$ and any complex $A^{*} \in C(\mathcal{A})$, the existence of an isomorphism $\operatorname{Hom}_{C(\mathcal{A})}\left(S^{0}(M), A^{*}\right) \cong \operatorname{Hom}_{\mathcal{A}}\left(M, Z^{0}\left(A^{*}\right)\right)$ which is natural in both variables is clear, that is, $Z^{0}$ is a right adjoint functor of $S^{0}$. It immediately follows that if $f: S^{0}(M) \rightarrow E^{*}$ is an $\mathcal{E}$-preenvelope in $C(\mathcal{A})$, then $Z^{0}(f): M \rightarrow Z^{0}\left(E^{*}\right)$ is a $\mathcal{Z}(\mathcal{E})$-preenvelope in $\mathcal{A}$.

b) Given any $M \in \mathcal{A}$, consider a special $\mathcal{E}$-preenvelope of $S^{0}(M)$,

$$
0 \longrightarrow S^{0}(M) \longrightarrow E^{*} \longrightarrow X^{*} \longrightarrow 0
$$

(so $X^{*} \in{ }^{\perp} \mathcal{E}$ ). Applying $Z^{0}(-)$ we get the exact sequence

$$
0 \longrightarrow M \longrightarrow Z^{0}\left(E^{*}\right) \longrightarrow Z^{0}\left(X^{*}\right) \longrightarrow 0,
$$

so we just need to prove that $Z^{0}\left(X^{*}\right) \in{ }^{\perp} \mathcal{Z}(\mathcal{E})$.

Let then $Z \in \mathcal{Z}(\mathcal{E})$ be any object and fix $\widehat{E}^{*} \in \mathcal{E}$ such that $Z^{0}\left(\widehat{E}^{*}\right)=Z$. Since $\widehat{E}^{0}$ is injective, the exactness of the sequence

$$
0 \longrightarrow Z \longrightarrow \widehat{E}^{0} \longrightarrow Z^{1}\left(\widehat{E}^{*}\right) \longrightarrow 0
$$

shows that $\operatorname{Ext}\left(Z^{0}\left(X^{*}\right), Z\right)=0$ if and only if

$$
\operatorname{Hom}_{\mathcal{A}}\left(Z^{0}\left(X^{*}\right), \widehat{E}^{0}\right) \stackrel{\operatorname{Hom}\left(Z^{0}\left(X^{*}\right), \delta^{0}\right)}{\longrightarrow} \operatorname{Hom}_{\mathcal{A}}\left(Z^{0}\left(X^{*}\right), Z^{1}\left(\widehat{E}^{*}\right)\right)
$$


is an epimorphism $\left(\delta^{0}: \widehat{E}^{*} \rightarrow Z^{1}\left(\widehat{E}^{*}\right)\right.$ being the $0^{t h}$-differential $)$.

Now, since $X^{n}=E^{n} \forall n \neq 0$, we have that $X^{n} \in \mathcal{Y} \forall n \neq 0$ and so that $X^{n} \in$ ${ }^{\perp} \mathcal{Z}(\mathcal{E}) \forall n \neq 0$. Thus, if $\alpha \in \operatorname{Hom}_{\mathcal{A}}\left(Z^{0}\left(X^{*}\right), Z^{1}\left(\widehat{E}^{*}\right)\right)$ is any morphism, applying standard arguments (noting that $\operatorname{Ext}_{\mathcal{A}}\left(X^{k}, Z^{k+1}\left(\widehat{E}^{*}\right)\right)=0 \forall k<0$ and that each $\widehat{E}^{n}$ is injective) we can lift $\alpha$ to a morphism of complexes $f: X^{*} \rightarrow \widehat{E}^{*}[1]$, where $\widehat{E}^{*}[1]$ stands for the one-degree suspension of $\widehat{E}^{*}$. In particular $Z^{0}(f)=\alpha$.

If we prove that $f$ is null-homotopic with $s_{0}: X^{0} \rightarrow \widehat{E}^{0}$ and $s_{1}: X^{1} \rightarrow \widehat{E}^{1}$ the $0^{t h}$ and $1^{\text {st }}$ parts of the homotopy, we would have that, if $k: Z^{0}\left(X^{*}\right) \rightarrow X^{0}$ is the inclusion, then $s \circ k \in \operatorname{Hom}_{\mathcal{A}}\left(Z^{0}\left(X^{*}\right), \widehat{E}^{0}\right)$ maps onto $\alpha$ via the morphism $\operatorname{Hom}\left(Z^{0}\left(X^{*}\right)\right.$, $\left.\delta^{0}\right)$. This would complete the proof.

Let us then see that $f$ is null-homotopic. If we let $\operatorname{Ext}_{C(\mathcal{A})}^{s s}\left(X^{*}, \widehat{E}^{*}\right)$ denote the group of semi-split, that is, componentwise split, exact sequences of complexes (which can also be found in the literature under the notation $\operatorname{Ext}_{d w}(-,-)$ ), we have $0=\operatorname{Ext}_{C(\mathcal{A})}^{s s}\left(X^{*}, \widehat{E}^{*}\right)=\operatorname{Ext}_{C(\mathcal{A})}\left(X^{*}, \widehat{E}^{*}\right)$. But it is known that $\operatorname{Ext}_{C(\mathcal{A})}^{s s}\left(X^{*}, \widehat{E}^{*}\right) \cong$ $\operatorname{Hom}_{\mathcal{K}(\mathcal{A})}\left(X^{*}, \widehat{E}^{*}[1]\right)$ where $\mathcal{K}(\mathcal{A})$ stands for the homotopy category, so we finally have that $\operatorname{Hom}_{\mathcal{K}(\mathcal{A})}\left(X^{*}, \widehat{E}^{*}[1]\right)=0$, that is, $f$ is null-homotopic.

c) If $M$ is any object of $\mathcal{A}$ and $f: S^{0}(M) \rightarrow E^{*}$ is an $\mathcal{E}$-envelope then we know $Z^{0}(f): M \rightarrow Z^{0}\left(E^{*}\right)$ is a $\mathcal{Z}(\mathcal{E})$-preenvelope.

Now, given a commutative diagram

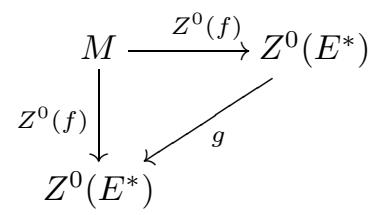

as in b), we can lift $g$ to a morphism of complexes $\bar{g}: E^{*} \rightarrow E^{*}$, and it is immediate to check that $\bar{g} f=f$, so being $f$ an $\mathcal{E}$-envelope means $\bar{g}$ is an automorphism. Then $g$ must be an automorphism.

\section{Gorenstein flat precovers in Grothendieck categories}

The main purpose of this section is to study the existence of Gorenstein flat precovers in locally finitely presented Grothendieck categories. But to define Gorenstein flat objects will shall need the use of a tensor product. Thus, we start by setting up what a tensor product will mean to us. Recall that, unless otherwise specified, all categories are supposed to be locally finitely presented Grothendieck categories. 
Definition 4.1. A tensor product on $\mathcal{B}$ and $\mathcal{A}$ is defined as a bifunctor $-\otimes-$ : $\mathcal{B} \times \mathcal{A} \rightarrow \mathcal{A} b$ that is right exact in each one of the two variables and preserves direct limits in each one of the two variables.

The concept of a flat object with respect to a given tensor product turns out to be quite natural now.

Definition 4.2. If $\mathcal{A}$ and $\mathcal{B}$ are two Grothendieck categories with a tensor product $-\otimes-: \mathcal{B} \times \mathcal{A} \rightarrow \mathcal{A} b$, an object $F$ in $\mathcal{A}$ (in $\mathcal{B}$ ) is said to be flat relative to $\otimes$ if the tensor functor $-\otimes F: \mathcal{B} \rightarrow \mathcal{A} b$ (the tensor functor $F \otimes-$ ) is exact.

We shall call these objects flat and will not specify the tensor product the object is relatively flat to, since usually there will be no confusion.

Definition 4.3. An object $M$ in $\mathcal{A}$ is said to be Gorenstein flat if it is the 0 -syzygy of an exact complex of flat objects in $\mathcal{A}$

$\mathbf{F}$ :

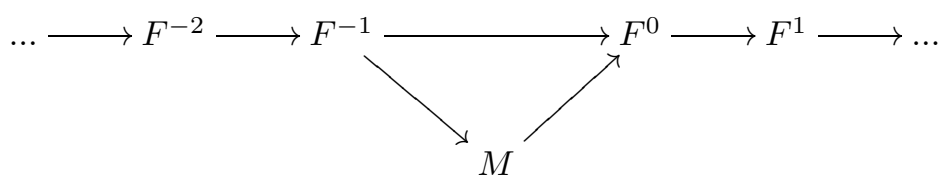

such that $E \otimes \mathbf{F}$ is exact for every injective object $E$ of $\mathcal{B}$.

We now mimic the construction of the tensor product of complexes of modules: if $B$ and $A$ are two complexes in $C(\mathcal{B})$ and $C(\mathcal{A})$ respectively, with differentials $d_{B}^{n}$ and $d_{A}^{n}$ respectively, the tensor product of $B$ and $A$ is defined as the complex $B \dot{\otimes} A$ given by

$$
(B \dot{\otimes} A)^{n}=\oplus_{t \in \mathbb{Z}} B^{t} \otimes A^{n-t}
$$

and differentials

$$
\begin{gathered}
d^{n}:(B \dot{\otimes} A)^{n} \longrightarrow(B \dot{\otimes} A)^{n+1} \\
d^{n}=\prod_{t}\left(d_{A}^{t} \otimes 1_{A^{n-t}}+(-1)^{t}\left(1_{B^{t}} \otimes d_{A}^{n-t}\right)\right)
\end{gathered}
$$

From this complex a new complex of abelian groups, $B \otimes A$, is then defined as

$$
(B \bigotimes A)^{n}=\frac{(B \dot{\otimes} A)^{n}}{\operatorname{Im}\left(d^{n-1}\right)}
$$

with differentials $\overline{d^{n}}=\prod_{t}\left(d_{B}^{t} \otimes 1_{A^{n-t}}\right)$.

It is known that every locally finitely presented Grothendieck category is equivalent to the full subcategory of flat objects of a category of modules over a ring with enough idempotents. Thus, having a tensor product on two such categories is the same as having a tensor product (in the sense of Definition 4.1) on the corresponding 
subcategories of flat modules, $\mathcal{F}(\operatorname{Mod}-S)$ and $\mathcal{F}(R$-Mod), and so having flat objects in our original categories and having flat objects in the categories $\mathcal{F}(R$-Mod) (that is, objects which are flat in $\mathcal{F}(R$-Mod), not just flat modules) is equivalent. We will make use of this equivalence without mentioning the identification, that is, unless otherwise specified, our categories in this section will always be the full subcategories of flat modules of the whole category of modules over a certain ring with enough idempotents.

Notation Along the rest of this section we will let $P$ be the direct sum of a copy of (up to isomorphism) every finitely generated complex in $C(\mathcal{B})$.

It is not hard to see that $-\dot{\otimes}-: C(\mathcal{B}) \times C(\mathcal{A}) \rightarrow C(\mathcal{A} b)$ is a bifunctor that preserves direct limits in each one of the two variables since $-\otimes-: \mathcal{B} \times \mathcal{A} \rightarrow \mathcal{A} b$ does. But then $-\otimes-: C(\mathcal{B}) \times C(\mathcal{A}) \rightarrow C(\mathcal{A} b)$ does preserves direct limits in both variables. Thus, with the same type of arguments as in [8, Lemma 3] we get the following.

Lemma 4.4. Given any cardinal number $\aleph$ with $|H| \leq \aleph$ there exists a cardinal number $\nu$ with the following property: for any complex $X$ and any subcomplex $L \leq X$ with $|L| \leq \aleph$, there is a subcomplex $L^{\prime} \leq X$ containing $L$ such that $\left|L^{\prime}\right| \leq \nu$ and that $\operatorname{ker}(P \otimes L \rightarrow P \otimes X)=\operatorname{ker}\left(P \otimes L \rightarrow P \otimes L^{\prime}\right)$.

Proof. Let us start by finding the complex $L^{\prime}$ with our desired property and then we will check out the cardinalities.

So, we first note that $X$ can be written as a direct union of finitely generated subcomplexes, $X=\cup_{i \in I} Y_{i}$. Thus, taking $X_{i}=Y_{i}+L \forall i$ we see that again $X$ is the direct union $X=\cup_{i \in I} X_{i}$ where the morphisms $k_{i j}: X_{i} \rightarrow X_{j}$ are the inclusions whenever $i \leq j$ and $k_{i}: X_{i} \rightarrow X$ are the inclusion morphisms too, but now $L$ is a subobject of every one of the $X_{i}$.

Now, let $k: L \rightarrow X$ denote the injection of complexes and choose any $z \in$ $\operatorname{ker}\left(1_{P} \otimes k\right)$. Thus, by the properties of the direct limits we know there is an index $i \in I$ and an element $x_{i} \in P \otimes X_{i}$ such that $\left(1_{P} \otimes k_{i}\right)\left(x_{i}\right)=\left(1_{P} \otimes k\right)(z)=0$.

If we call $\lambda_{i}: L \rightarrow X_{i}$ the inclusion morphism then we see that $\left(1_{P} \otimes k_{i}\right)\left(1_{P} \otimes\right.$ $\left.\lambda_{i}\right)(z)=\left(1_{P} \otimes k\right)(z)=0$, so again, by the properties of the direct limits we can find $i_{z} \geq i$ such that $\left(1_{P} \otimes k_{i i_{z}}\right)\left(1_{P} \otimes \lambda_{i}\right)(z)=0$. But $\left(1_{P} \otimes k_{i i_{z}}\right)\left(1_{P} \otimes \lambda_{i}\right)(z)=\left(1_{P} \otimes \lambda_{i_{z}}\right)(z)$, so we see that indeed $z \in \operatorname{ker}\left(1_{P} \otimes \lambda_{i z}\right)$.

Let us call $L^{\prime}=\sum_{z \in \operatorname{ker}\left(1_{P} \otimes k\right)} X_{i_{z}}$ and let $k^{\prime}: L \rightarrow L^{\prime}$ and $\delta_{i_{z}}: X_{i_{z}} \rightarrow L^{\prime}$ be the inclusion morphisms. Then, for any $z \in \operatorname{ker}\left(1_{P} \otimes k\right)$ we have $\left(1_{P} \otimes k^{\prime}\right)(z)=\left(1_{P} \otimes\right.$ $\left.\delta_{i_{z}}\right)\left(1_{P} \otimes \lambda_{i_{z}}\right)(z)=0$, so we have that $\operatorname{ker}\left(1_{P} \otimes k\right) \subseteq \operatorname{ker}\left(1_{P} \otimes k^{\prime}\right)$, and the converse is clear.

Of course $L \leq L^{\prime}$ so it only remains to find a cardinal number $\nu$ such that: a) $\left|L^{\prime}\right| \leq \nu$, b) $\nu$ depends on the choice of $\aleph$ (in the sense that $|H| \leq \aleph$ and that 
$|L| \leq \aleph$ ), and c) $\nu$ is independent of the choice of $X$ and $L$ themselves (although the cardinality of $L$ must be $\leq \aleph)$.

By Proposition 2.3 we know there is a set of representatives of all complexes $L$ of cardinality $\leq \aleph$, so we can find the cardinal

$$
\mu=\sup \left\{\left|\operatorname{ker}\left(1_{P} \bigotimes k_{L}\right)\right| ;|L| \leq \aleph\right\}
$$

being $k_{L}: L \rightarrow X$ the corresponding injection. Note that no matter where $k_{L}$ goes to, that is, no matter what complex we see $L$ embedded in, $\operatorname{ker}\left(1_{P} \otimes k_{L}\right)$ is an abelian subgroup of $P \otimes L$ and so $\left|\operatorname{ker}\left(1_{P} \otimes k_{L}\right)\right| \leq|P \otimes L|$. In other words, the choice of $\mu$ does not depend on the complex $X$ nor on its cardinality.

Now, we know by 2.10 that there is a cardinal number $\lambda$ which depends on $\aleph$ $(|H| \leq \aleph)$ that upper bounds the cardinality of every finitely generated object $Y$, so if $L \leq X$ is any subobject with $|L| \leq \aleph$, an application of Proposition 2.9 gives us a cardinal number $\alpha$ such that $|L+Y| \leq \alpha$ for every finitely generated subobject $Y$ of $X$, independently of who $X$ or $L$ are. This applies to our subcomplexes $X_{i}$, so we see that $\left|X_{i}\right| \leq \alpha \forall i$.

Then, another application of Proposition 2.9 gives us the cardinal number $\nu$ which we are looking for.

Let us now define the concept of tpurity.

Definition 4.5. If $A$ is any object in $\mathcal{A}$, a subobject $A^{\prime} \leq A$ is said to be tpure whenever $B \otimes A^{\prime} \rightarrow B \otimes A$ is a monomorphism for every $B \in \mathcal{B}$. In this case we will say that the sequence $0 \rightarrow A^{\prime} \rightarrow A \rightarrow A / A^{\prime} \rightarrow 0$ is tpure.

A subcomplex $A^{\prime} \leq A \in C(\mathcal{A})$ will be said to be tpure provided that it is tpure with respect to the tensor $\otimes$.

Since $\otimes$ commutes with direct limits, we see that tpurity of monomorphisms in $C(\mathcal{A})$ can be checked out by just testing with $P \otimes-$.

Proposition 4.6. For any cardinal number $\aleph$ with $|H| \leq \aleph$, there is a cardinal number $\nu$ such that for every complex $X$ in $C(\mathcal{A})$ and every subcomplex $L$ of cardinality $\leq \aleph$ we can find a tpure subcomplex $S \leq X$ of cardinality $\leq \nu$ containing $L$.

Proof. By Lemma 4.4 we get a cardinal number $\nu_{0}$ and a subcomplex $S_{0} \leq$ $X$ of cardinality $\leq \nu_{0}$ containing $L$ such that $\operatorname{ker}(P \otimes L \rightarrow P \otimes X)=\operatorname{ker}(P \otimes L \rightarrow$ $\left.P \otimes S_{0}\right)$.

Apply again Lemma 4.4 and find another cardinal number $\nu_{1}$ and a subcomplex $S_{1} \leq X$ of cardinality $\leq \nu_{1}$ containing $S_{0}$ and satisfying $\operatorname{ker}\left(P \otimes S_{0} \rightarrow P \otimes X\right)=$ $\operatorname{ker}\left(P \otimes S_{0} \rightarrow P \bigotimes S_{1}\right)$. 
Repeat this argument finding a chain of $S_{n}, n \geq 0$, with $\left|S_{n}\right| \leq \nu_{n} \forall n$, and construct $S$, the complex generated by all $S_{n}$.

It is worth noting here that Lemma 4.4 guarantees that $\nu_{0}$ only depends on $\aleph$, that is, no matter what the subcomplex $L \leq X$ is (provided that $|L| \leq \aleph$ ) the cardinal number $\nu_{0}$ can always be chosen the same. Similarly, $\nu_{1}$ only depends on $\nu_{0}$ (so actually it only depends on $\aleph$ ), and so on.

Thus, an application of Proposition 2.7 gives us a cardinal number $\nu$ which in the end only depends on $\aleph$ (and not on $L$ ), such that $|S| \leq \nu$.

It only remains to prove the tpurity of $S$.

Let us call $\alpha_{n, n+1}: S_{n} \rightarrow S_{n+1}, \xi_{n}: S_{n} \rightarrow S=\lim _{\rightarrow} S_{n}$ and $k_{n}: S_{n} \rightarrow X$ the injections. Then we have the commutativity of the following diagrams
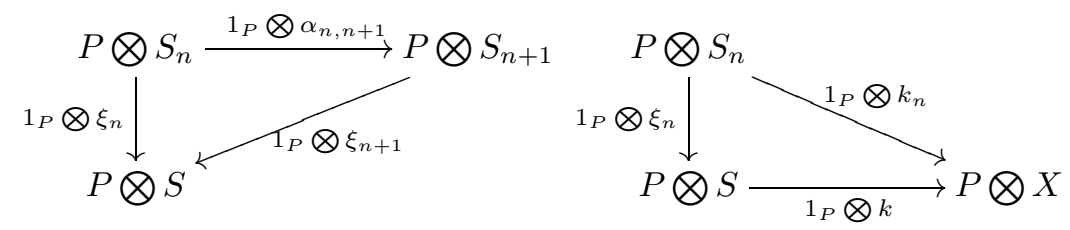

for every natural $n$.

If $x \in P \otimes S$ then there are $n$ and $x_{n} \in P \otimes S_{n}$ such that $\left(1_{P} \otimes \xi_{n}\right)\left(x_{n}\right)=$ $x$. If, in addition, $x \in \operatorname{ker}\left(1_{P} \otimes k\right)$ then $\left(1_{P} \otimes k_{n}\right)\left(x_{n}\right)=0$. But by construction $\operatorname{ker}\left(1_{P} \otimes k_{n}\right)=\operatorname{ker}\left(1_{P} \bigotimes \alpha_{n, n+1}\right)$, so we have

$$
0=\left(1_{P} \bigotimes \xi_{n+1}\right)\left(1_{P} \bigotimes \alpha_{n, n+1}\right)\left(x_{n}\right)=\left(1_{P} \bigotimes \xi_{n}\right)\left(x_{n}\right)=x .
$$

In other words $1_{P} \otimes k$ is a monomorphism.

The next result is an adaptation to our setting of [13, Proposition 2.3.24].

Proposition 4.7. Let $\aleph$ be a cardinal number such that $|H| \leq \aleph$. Then there is a cardinal number $\nu$ such that for any exact complex $X$ and every subcomplex $L \leq X$ of cardinality $\leq \aleph$ we can find an exact subcomplex $E \leq X$ containing $L$ with cardinality $|E| \leq \nu$.

Proof. Any element $x \in Z(L)$ is a boundary in $X$ since $X$ is exact so there is some $y_{x} \in B(X)$ such that $d\left(y_{x}\right)=x$. Let $S_{0}=L$ and construct

$$
S_{1}=S_{0}+\sum_{x \in Z(L)}<y_{x}>.
$$

It is clear by construction that $Z\left(S_{0}\right) \subseteq B\left(S_{1}\right)$, and about cardinalities we have $\left|S_{0}\right| \leq \aleph$ so $|Z(L)| \leq \aleph(Z(L) \leq L)$, and $\left|<y_{x}>\right| \leq \lambda$ for any $x \in Z(L)$ ( $\lambda$ is the cardinal number found in Proposition 2.10). Thus, we find a cardinal number $\nu_{1}$ such 
that $\left|S_{1}\right| \leq \nu_{1}$ (see Proposition 2.9). The important fact about this $\nu_{1}$ is that it is independent of the choice of $L$ and $X$.

Repeat this argument to find a subcomplex $S_{2} \leq X$ containing $S_{1}$ such that $Z\left(S_{1}\right) \subseteq B\left(S_{2}\right)$ and that $\left|S_{2}\right| \leq \nu_{2}$ for some $\nu_{2}$ depending only on $\nu_{1}$.

Following this way we find a chain $S_{0} \subseteq S_{1} \subseteq \ldots$ of subcomplexes of $X$ with $\left|S_{n}\right| \leq \nu_{n}$ and $Z\left(S_{n}\right) \subseteq B\left(S_{n+1}\right)$ for every $n$. If we then consider the sum $E$ of all of them we clearly see that $E$ is an exact subcomplex of $X$ containing $L$.

Now, Proposition 2.7 guarantees the existence of a cardinal number $\nu$ that depends on the family of cardinals $\left\{\nu_{n} ; n \in \mathbb{N}\right\}$ but that is independent of the choice of $L$ or $X$, such that $|E| \leq \nu$.

Combining Propositions 4.6 and 4.7 we get the following.

Proposition 4.8. For any cardinal number $\aleph$ with $|H| \leq \aleph$, there is a cardinal number $\nu$ such that for every exact complex $X$ in $C(\mathcal{A})$ and every subcomplex $L$ of cardinality $\leq \aleph$ we can find an exact and tpure subcomplex of $X$ of cardinality $\leq \nu$ containing $L$.

Proof. By Proposition 4.6 we find a cardinal number $\nu_{1}$ and a tpure subcomplex $T_{1} \leq X$ containing $L$ of cardinality $\leq \nu_{1}$. Now apply Proposition 4.7 to $T_{1}$ to get a cardinal number $\nu_{2}$ and an exact subcomplex $E_{2}$ of $X$ containing $T_{1}$ of cardinality $\leq \nu_{2}$. Again, $\nu_{1}$ only depends on $\aleph$ and $\nu_{2}$ only depends on $\nu_{1}$, that is, we can always choose $\nu_{2}$ at this step independently of the choice of $L$ and $X$. Apply again Proposition 4.6 to $E_{2}$ and keep repeating this "zig-zag" argument to find a chain of subcomplexes of $X$ whose direct union clearly: is exact (direct limits of exact complexes are always exact), is tpure (direct limits of tpure complexes are tpure since $\otimes$ commutes with direct limits), contains our original complex $L$ and, has cardinality $\leq \nu$ for some cardinal number $\nu$ which we get by just applying Proposition 2.7, and which is independent of the choice of $L$ and $X$.

Now we prove that given any object $C \in \mathcal{B}$, any tpure subcomplex of a $C \otimes$-exact complex is again $C \otimes$-exact.

Proposition 4.9. If $C$ is any object in $\mathcal{B}$ and $X \in C(\mathcal{A})$ is any $C \otimes-$ exact complex, then any tpure subcomplex $L \leq X$ is again $C \otimes-$ exact.

Proof. It is clear that $C \otimes M=S^{0}(C) \dot{\otimes} M$ for any complex $M \in C(\mathcal{A})$ so if $y \in$ $C \otimes L$ is any cycle then $y$ is a cycle (and so a boundary) in $C \otimes X=S^{0}(C) \dot{\otimes} X$. Thus $y$ is zero in $S^{0}(C) \otimes X$ by construction and then $y$ must be zero in $S^{0}(C) \otimes L$ since $S^{0}(C) \otimes L \rightarrow S^{0}(C) \otimes X$ is a monomorphism. It then follows that $y$ is a boundary in $S^{0}(C) \dot{\otimes} L=C \otimes L$. 
In [13, Proposition 4.2.1] it is shown that for any complex $C$ and any object $M, D^{0}(M) \otimes C \cong M \otimes C$. This isomorphism is natural in both variables. It is then clear that if $L$ is a tpure subcomplex of $X \in C(\mathcal{A})$ then every component $L^{n}$ is tpure in $X^{n}$. But then, if every $X^{n}$ is flat we get that every $L^{n}$ and every $X^{n} / L^{n}$ are flat. Thus we have the following:

Corollary 4.10. If $C \in \mathcal{B}, X \in C(\mathcal{A})$ is $C \otimes-$ exact and $X^{n}$ is flat for every $n$, then, for every tpure subcomplex $L \leq X$ the following assertions hold:

1) $L$ is $C \otimes-$ exact.

2) $L^{n}$ is flat for every $n$.

3) $X / L$ is $C \otimes-$ exact.

4) $X^{n} / L^{n}$ is flat for every $n$.

The concept of $\mathcal{C}$-filtration of an object has played a key role in the development of the theory of covers and envelopes in the last years. It can be found, for instance, in [12, Definition 2.1]. We state it here for completeness.

Definition 4.11. Given a class of objects $\mathcal{C}$, an object $X \in O b(\mathcal{A})$ is said to be $\mathcal{C}$-filtered if there is an ordinal number $\varepsilon$ and a continuous chain $\left\{C_{\alpha} ; \alpha \leq \varepsilon\right\}$ of subobjects of $X$ (so $C_{0}=0$ and $C_{\mu}=\cup_{\alpha<\mu} C_{\alpha}$ for every limit ordinal $\mu \leq \varepsilon$ ) such that $C_{\varepsilon}=M$ and $C_{\alpha+1} / C_{\alpha} \in \mathcal{C} \forall \alpha$.

Let us now call $\mathcal{E} \mathcal{F}$ the class of all exact complexes in $C(\mathcal{A})$ whose components are all flat and such that they remain exact after applying the functor $E \otimes-$ for every injective object $E$ of $\mathcal{B}$. Choose a representative of any complex in $\mathcal{E} \mathcal{F}$ of cardinality $\leq \aleph$. Call $\mathcal{E F}^{\aleph}$ the set of all these representatives.

Theorem 4.12. Let $\aleph$ be any cardinal number such that $|H| \leq \aleph$ and consider the cardinal number $\nu$ given in Proposition 4.8. Then every object of the class $\mathcal{E} \mathcal{F}$ is $\mathcal{E F}^{\nu}$-filtered.

Proof. Given any complex $X \in \mathcal{E} \mathcal{F}$ choose any element $x \in X$ and find an exact and tpure subcomplex $S_{1} \leq X$ of cardinality $\leq \nu$ containing $x$. By Corollary 4.10 both $S_{1}$ and $X / S_{1}$ are in $\mathcal{E} \mathcal{F}$ (so indeed $S_{1} \in \mathcal{E} \mathcal{F}^{\nu}$ ), so we can choose any $y \in X / S_{1}$ and find an exact and tpure subcomplex $S_{2} / S_{1} \leq X / S_{1}$ containing $y$ of cardinality $\leq \nu$. Then $S_{2} / S_{1} \in \mathcal{E} \mathcal{F}^{\nu}$ and $X / S_{2} \in \mathcal{E F}$ so we can repeat the process letting $S_{\omega_{0}}=\lim _{\rightarrow} S_{n}$.

Every $S_{i}$ is exact so $S_{\omega_{0}}$ is exact too. Similarly, every $S_{i}$ is $E \otimes-$ exact (for every injective $E$ ), so again $S_{\omega_{0}}$ is $E \otimes-$ exact (for every injective $E$ ) since $E \otimes-$ commutes with direct limits in $\mathcal{A}$.

Finally, we can see by induction that since $S_{i}^{n}, S_{i+1}^{n} / S_{i}^{n}$ are flat, $S_{i+1}^{n}$ is flat too. Therefore $S_{\omega_{0}}^{n}$ is again flat for every $n$.

We then have $S_{\omega_{0}} \in \mathcal{E} \mathcal{F}$, so we can choose any element and proceed as before.

This means every complex in $\mathcal{E} \mathcal{F}$ is $\mathcal{E F}^{\nu}$-filtered. 
At this point, the existence of $\mathcal{E} \mathcal{F}$-precovers in the category $C(\mathcal{A})$ can be proved under certain conditions.

Theorem 4.13. If the class of flat objects of $\mathcal{A}$ is closed under extensions, then every complex in $C(\mathcal{A})$ has an $\mathcal{E} \mathcal{F}$-precover.

Proof. By [25, Corollary 2.15], the existence of $\mathcal{E} \mathcal{F}$-precovers would be guaranteed if we see that the class $\mathcal{E F}$ coincide with the class of all $X$-filtered complexes for some set $X$, that is, $\mathcal{E} \mathcal{F}=X$-filtered. But by Theorem 5.3 every complex in $\mathcal{E} \mathcal{F}$ is $\mathcal{E} \mathcal{F}^{\nu}$-filtered, that is, $\mathcal{E} \mathcal{F} \subseteq \mathcal{E} \mathcal{F}^{\nu}$-filtered, so we just need to prove the converse inclusion.

Let then $\omega$ be an ordinal number and $\left\{S_{\alpha} ; \alpha \leq \omega\right\}$ be an $\mathcal{E F}^{\nu}$-filtration. Let us prove that $S_{\omega} \in \mathcal{E} \mathcal{F}$.

Since $S_{\alpha+1} / S_{\alpha} \in \mathcal{E} \mathcal{F}^{\nu}$ for every $\alpha$ and $S_{0}=0$, we have that $S_{1} \in \mathcal{E} \mathcal{F}^{\nu}$. This means (in particular) that $S_{1}$ is exact, and since $S_{2} / S_{1} \in \mathcal{E} \mathcal{F}^{\nu}, S_{2} / S_{1}$ is exact too. But exact complexes are closed under extensions, so we see that $S_{2}$ is exact. This means that $S_{n}$ is exact for every positive integer $n$, so, since direct limits of exact complexes are exact, $S_{\omega_{0}}$ is exact. It is clear now that a transfinite induction process proves that $S_{\omega}$ is indeed exact.

Now, flat objects of $\mathcal{A}$ are closed under direct limits, and by the hypotheses, they are closed under extensions too, so the same transfinite induction process gives us that every component of $S_{\omega}$ is flat.

Finally, to prove the $(E \otimes-)$-exactness of $S_{\omega}$ we will use again this transfinite induction process. The limit part is immediate since, as before, direct limits of exact complexes are exact, so the problem reduces to prove that, being $S_{1}$ and $S_{2} / S_{1}(E \otimes-)$-exact complexes, $S_{2}$ is $(E \otimes-)$-exact too.

Let us denote by $\delta_{1}, \delta_{2}$ and $\delta_{3}$ the differentials of $S_{1}, S_{2}$ and $S_{2} / S_{1}$ respectively, and let $C_{i}^{n}=\operatorname{coker}\left(\delta_{i}^{n-1}\right), K_{i}^{n}=\operatorname{ker}\left(\delta_{i}^{n}\right), i=1,2,3$.

$S_{1}$ is exact and $(E \otimes-)$-exact, so $E \otimes C_{1}^{n}=\operatorname{coker}\left(1_{E} \otimes \delta_{1}^{n-1}\right)=\operatorname{ker}\left(1_{E} \otimes \delta_{1}^{n}\right)=E \otimes$ $K_{i}^{n}$ for every positive integer $n$, and the same happens to $S_{2} / S_{1}$.

On the other hand, since $0 \rightarrow S_{1} \rightarrow S_{2} \rightarrow S_{2} / S_{1} \rightarrow 0$ is exact and each of the three complexes is exact, an easy application of the Snake Lemma gives that the induced sequence on the cokernels, $0 \rightarrow C_{1} \rightarrow C_{2} \rightarrow C_{3} \rightarrow 0$ is also exact. This means that, applying $E \otimes-$, we get an exact sequence

$$
E \otimes C_{1} \longrightarrow E \otimes C_{2} \longrightarrow E \otimes C_{3} \longrightarrow 0
$$


Thus, we have a commutative diagram

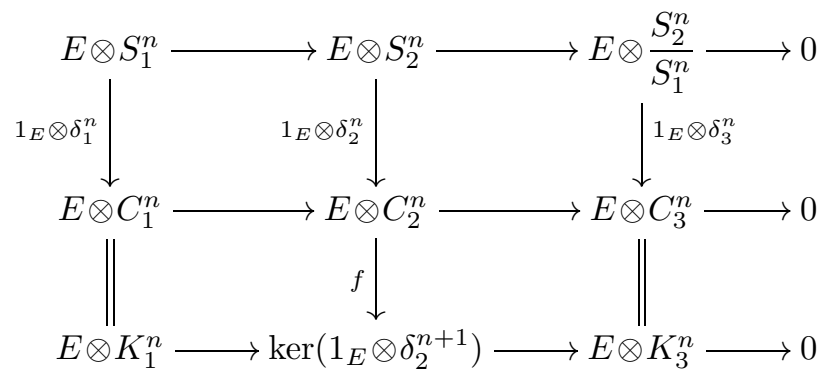

with exact rows where $1_{E} \otimes \delta_{i}^{n}$ are all epimorphisms and $f$ is a monomorphism.

Then, the four lemma says that $f \circ\left(1_{E} \otimes \delta_{2}^{n}\right)$, and so $f$, is an epimorphism. We then see that $f$ is indeed an isomorphism and so that $E \otimes S_{2}$ is exact.

It is now worth noting that recently, a paper by Estrada and Gillespie in which the authors prove a similar result to Theorem 4.12, and so to Theorem 4.13 (see [11, Theorem 3.7, Corollary 3.11 and Theorem 6.5]), has appeared.

Now, the main result of this section follows using Proposition 3.1.

Theorem 4.14. For any two locally finitely presented Grothendieck categories $\mathcal{A}$ and $\mathcal{B}$ and for any tensor product $\otimes: \mathcal{B} \times \mathcal{A} \rightarrow \mathcal{A} b$, the class of Gorenstein flat objects in $\mathcal{A}$ with respect to this tensor product is precovering provided that the class of flat objects of $\mathcal{A}$ is closed under extensions.

\section{Gorenstein injective preenvelopes}

The main subject of this section is the study of the existence of Gorenstein injective preenvelopes in Grothendieck categories. We will prove (Theorem 5.4) that, over any locally noetherian Grothendieck category, every object has a Gorenstein injective preenvelope if the class of all Gorenstein injective objects is closed under direct products.

We will make use of a slight variation of what we know as Kaplansky classes. Kaplansky classes have been widely studied and proved to be very useful when establishing the existence of preenvelopes. They are defined in terms of a given cardinal number. However, defining them by means of a single cardinal number is not completely useful for our purposes. Thus, we now give the following extension of the concept.

Definition 5.1. A class of objects $\mathcal{F}$ of $\mathcal{A}$ will be said to be pseudo Kaplansky provided that for any cardinal number $\lambda$ there exists a cardinal number $\aleph$ such that 
given any object $O \in \mathcal{F}$ and any subobject $S \leq O$ of cardinality $|S|<\lambda$, there exists $F \in \mathcal{F}$ of cardinality $|F|<\aleph$ such that $S \leq F \leq O$.

But why are pseudo Kaplansky classes interesting when finding preenvelopes? Here we give a result that clarifies such a question.

Theorem 5.2. Let $\mathcal{F}$ be a pseudo Kaplansky class. If $\mathcal{F}$ is closed under direct products, then it is preenveloping. When $\mathcal{F}$ is closed under direct summands, the converse is also true.

Proof. Let $A$ be any object of $\mathcal{A}$, call $\varkappa=|A|$, let $\lambda$ be the cardinal number given in Proposition 2.5, and let $\aleph$ be the cardinal number obtained from $\lambda$ in Definition 5.1. Consider a set $X$ of representatives of all the objects $F \in \mathcal{F}$ with $|F|<\aleph$ and let $\bar{F}=\prod_{F \in X} F^{\operatorname{Hom}(A, F)}$. Then there exists a unique morphism $\varphi: A \rightarrow$ $\bar{F}$ such that $\pi_{f, F} \circ \varphi=f$ for any $F \in X$ and any $f \in \operatorname{Hom}(A, F)$, where $\pi_{f, F}$ is the canonical projection.

Let $F_{1} \in \mathcal{F}$ be any object and $f: A \rightarrow F_{1}$ be any morphism.

Since $|\operatorname{Im}(f)|<\lambda$ there is a subobject $F_{2} \leq F_{1}$ of cardinality $\left|F_{2}\right|<\aleph$ containing $\operatorname{Im}(f)$ and such that $F_{2} \in \mathcal{F}$. Then $F_{2}$ is isomorphic to some $F \in X$ (let $g: F_{2} \rightarrow F$ be such an isomorphism). Then we have a commutative diagram

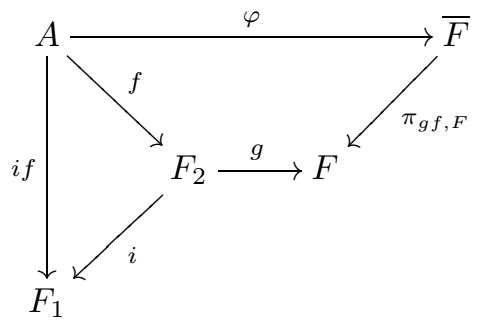

where $i$ is the injection. Thus, the morphism $i g^{-1} \pi_{g f, F}: \bar{F} \rightarrow F_{1}$ makes the outer diagram commutative. Since $\bar{F} \in \mathcal{F}$ by the hypotheses we see that $\varphi$ is an $\mathcal{F}$-preenvelope of $A$.

For the converse suppose that $\mathcal{F}$ is preenveloping and closed under direct summands. Then, if $\left\{F_{i} ; i \in I\right\}$ is a family of objects of $\mathcal{F}$, take the direct product $\prod_{i} F_{i}$ and a preenvelope $\varphi: \prod_{i} F_{i} \rightarrow F$.

Since $\varphi$ is an $\mathcal{F}$-preenvelope, the canonical projections $p_{i}: \prod_{i} F_{i} \rightarrow F_{i}$ induce a family of morphisms $\left\{f_{i}: F \rightarrow F_{i}\right\}$ such that $f_{i} \varphi=p_{i} \forall i$. But then this family induce a unique $f: F \rightarrow \prod_{i} F_{i}$ such that $p_{i} f=f_{i} \forall i$.

It is immediate now that $p_{i} f \varphi=p_{i} \forall i$ and so that $f \varphi=1_{\prod_{i} F_{i}}$. But $\mathcal{F}$ is closed under direct summands so we see that $\prod_{i} F_{i}$ is an object of $\mathcal{F}$. 
We now prove that the class of Gorenstein injective objects is always pseudo Kaplansky in every locally noetherian Grothendieck category. The proof of this fact is an adaptation of [9, Theorem 11.9.6] to or our general categorical setting and to the new concept of pseudo Kaplansky class. We give a complete proof here for completeness and to fix some typing bugs detected in the writing in [9].

Theorem 5.3. The class of all Gorenstein injective objects of a locally noetherian Grothendieck category is pseudo Kaplansky.

Proof. Suppose $\mathcal{A}$ is now locally noetherian and let $\gamma$ be any cardinal number, $M$ any Gorenstein injective object and $\ldots \rightarrow E^{-1} \stackrel{\delta^{-1}}{\rightarrow} E^{0} \stackrel{\delta^{0}}{\rightarrow} E^{1} \rightarrow \ldots$ a $\operatorname{Hom}(\mathcal{I} n j,-)$ exact exact sequence of injective objects with $M=\operatorname{ker}\left(\delta^{0}\right)$.

Given any subobject $S \leq M$ of cardinality $|S| \leq \gamma$, we will use induction to construct an exact and Hom-exact complex of injectives such that the kernel, $K^{0}$, of the zero derivation of this new complex (which is Gorenstein injective by definition) contains $S$ and is contained in $M$. In this induction process we will find the cardinal number $\aleph$ such that $\left|K^{0}\right| \leq \aleph$ and which will be independent on the choice of $M$ and $S$ (of course, as long as $|S| \leq \gamma$ ).

This induction process will be split into several induction arguments. First we will use a zig-zag induction procedure to construct an exact complex of injective objects that might not be Hom-exact. Then, from this last complex we will construct a new one which could now be not exact but that will be Hom-exact. Again, using this last complex we will find an exact (and perhaps not Hom-exact) new complex of injectives, and so on. Thus, at the end, the direct limit of all these complexes will be an exact and Hom-exact complex of injectives whose zero derivation will be the $K^{0}$ mentioned above.

So, to start, given $\gamma$ and $S \leq M$ with $|S| \leq \gamma$, we know by Proposition 2.2 that there is a cardinal number $\varkappa_{0}$ such that if $S \leq M$ is any subobject with $|S|<\gamma$ then there exists $S^{-1} \leq E^{-1}$ of cardinality less than or equal to $\varkappa_{0}$ such that $\delta^{-1}\left(S^{-1}\right)=S$. But by Proposition 2.8 we know there is a cardinal number $\nu_{0}$ such that $|Y| \leq \varkappa_{0} \Rightarrow$ $|E(Y)| \leq \nu_{0}$ no matter what the object $Y$ is. Therefore we have $\left|E\left(S^{-1}\right)\right| \leq \nu_{0}$. Let us call $I^{-1}=E\left(S^{-1}\right)$.

Now, using Proposition 2.5 we find another cardinal number $\nu_{1}$ such that $\left|\delta^{-1}\left(I^{-1}\right)\right| \leq \nu_{1}$ and so we know there is $\nu_{2}$ with $\left|I^{0}\right| \leq \nu_{2}$ (again $I^{0}=E\left(\delta^{-1}\left(I^{-1}\right)\right)$ ). Then we get a $\nu_{3}$ with $\left|\delta^{0}\left(I^{0}\right)\right| \leq \nu_{3}$ and so a $\nu_{4}$ with $\left|I^{1}\right| \leq \nu_{4}$.

We now turn over and go back: since $\left|I^{1} \cap \operatorname{Im}\left(\delta^{0}\right)\right| \leq \nu_{4}$ by Proposition 2.4, we can find a $D^{0} \leq E^{0}$ containing $I^{0}$ and of cardinality $\left|D^{0}\right| \leq \nu_{5}$ (for some $\nu_{5}$ ) such that $\delta^{0}\left(D^{0}\right)=I^{1} \cap \operatorname{Im}\left(\delta^{0}\right)$, and then (applying twice Proposition 2.5 and taking a supremum) there exists $\nu_{6}$ such that, if we call $A^{0}=E\left(D^{0}\right)$ and $A^{1}=E\left(\delta^{0}\left(A^{0}\right)\right)$, we have $\left|A^{0}\right| \leq \nu_{6}$ and $\left|A^{1}\right| \leq \nu_{6}$. Consider now $A^{0} \cap \operatorname{Im}\left(\delta^{-1}\right)$ and find $D^{-1} \leq E^{-1}$ 
containing $I^{-1}$, of cardinality $\left|D^{-1}\right| \leq \nu_{7}$, such that $\delta^{-1}\left(D^{-1}\right)=A^{0} \cap \operatorname{Im}\left(\delta^{-1}\right)$ and take the injective envelope $A^{-1}=E\left(D^{-1}\right)$. Thus, we can find a cardinal number $\nu_{8}$ such that all $A^{-1}, B^{0}=E\left(\delta^{-1}\left(A^{-1}\right)\right)$ and $B^{1}=E\left(\delta^{0}\left(A^{0}\right)\right)$ have cardinality $\leq \nu_{8}$. Give one more step back finding $D^{-2} \leq E^{-2}$ such that $\delta^{-2}\left(D^{-2}\right)=A^{-1} \cap \operatorname{Im}\left(\delta^{-2}\right)$, and consider its injective envelope $T^{-2}$. Again, there is some $\nu_{9}$ such that all $T^{-2}, T^{-1}=E\left(\delta^{-2}\left(T^{-2}\right)\right), T^{0}=E\left(\delta^{-1}\left(T^{-1}\right)\right)$ and $T^{1}=E\left(\delta^{0}\left(T^{0}\right)\right)$ have cardinality $\leq \nu_{9}$.

Now turn over again and go forward 4 steps following the arguments above, then turn back giving 5 steps, and continue this zig-zag process going $n$ steps forward, then $n+1$ steps backwards, then $n+2$ steps forward, and so on.

We get a direct system of inclusions which direct limit is an exact complex of injective objects (since all components of the complexes in the process are injective and the category is locally noetherian)

$$
H^{*}: \ldots \longrightarrow H^{-1} \longrightarrow H^{0} \longrightarrow H^{1} \longrightarrow \ldots
$$

such that $S \subseteq \operatorname{ker}\left(H^{0} \rightarrow H^{1}\right)$, and using Proposition 2.7 we see that $\left|H^{i}\right|<\nu, \forall i$ for some cardinal number $\nu$.

Let $X$ be a set of representatives of all indecomposable injective objects of $\mathcal{A}$ and let $E=\oplus_{I \in X} I$.

The complex

$$
\ldots \longrightarrow \operatorname{Hom}\left(E, H^{-1}\right) \stackrel{g^{-1}}{\longrightarrow} \operatorname{Hom}\left(E, H^{0}\right) \stackrel{g^{0}}{\longrightarrow} \operatorname{Hom}\left(E, H^{1}\right) \longrightarrow \ldots
$$

may not be exact, so for instance $\operatorname{Im} g^{-1} \neq \operatorname{ker} g^{0}$, but for every $\alpha \in \operatorname{ker} g^{0}$ there is a $\beta_{\alpha} \in \operatorname{Hom}\left(E, E^{-1}\right)$ such that $\delta_{*}^{-1}\left(\beta_{\alpha}\right)=\alpha$ since the complex

$$
\ldots \longrightarrow \operatorname{Hom}\left(E, E^{-1}\right) \stackrel{\delta_{*}^{-1}}{\longrightarrow} \operatorname{Hom}\left(E, E^{0}\right) \stackrel{\delta_{*}^{0}}{\longrightarrow} \operatorname{Hom}\left(E, E^{1}\right) \stackrel{\delta_{*}^{1}}{\longrightarrow} \ldots
$$

is exact. Moreover, if we call $\lambda=|E|$, then we know there is a cardinal number $\omega$ such that $\left|\operatorname{Im}\left(\beta_{\alpha}\right)\right| \leq \omega$ for any such $\alpha$ (Proposition 2.5) so $\left|\oplus_{\alpha \in \operatorname{ker} g^{0}} \operatorname{Im}\left(\beta_{\alpha}\right)\right| \leq \omega^{\left|\operatorname{ker} g^{0}\right|}$ by Proposition 2.6, and again (Proposition 2.9) there is a cardinal $\mu$ such that $\left|\sum_{\alpha \in \operatorname{ker} g^{0}} \operatorname{Im}\left(\beta_{\alpha}\right)\right| \leq \mu$. Therefore, the object $A^{-1}=H^{-1}+\sum_{\alpha \in \operatorname{ker} g^{0}} \operatorname{Im}\left(\beta_{\alpha}\right)$ has cardinality $\left|A^{-1}\right|<\eta_{0}$ (for some $\eta_{0}$ ).

Thus, the injective envelope $P^{-1}=E\left(A^{-1}\right) \leq E^{-1}$ is such that $|P|<\eta_{2}$ (for some $\left.\eta_{2}\right)$, and that $\operatorname{ker}\left(g^{0}\right) \subseteq \operatorname{Im}\left(\left.\delta_{*}^{-1}\right|_{\operatorname{Hom}\left(E, P^{-1}\right)}\right)$. Consider now the injective envelope $P^{0}=E\left(\delta^{-1}\left(P^{-1}\right)\right)$ and the corresponding $\eta_{3}$ with $\left|P_{0}\right| \leq \eta_{3}$. Then take the image $\delta^{0}\left(P^{0}\right)$ and enlarge it to its injective envelope $P^{1}$ (again with the corresponding $\left.\eta_{4}\right)$.

Now we start another zig-zag procedure as before, so we turn back and find $\eta_{5}$ and $C^{0} \leq P^{0}$ with $\delta^{0}\left(C^{0}\right)=P^{1} \cap \operatorname{Im}\left(\delta^{0}\right)$ and $\left|C^{0}\right| \leq \eta_{5}$. Then call $Q^{0}=E\left(C^{0}\right)$, 
$Q^{1}=E\left(\delta^{0}\left(Q^{0}\right)\right)$ and find $\eta_{6}$ with $\left|Q^{0}\right| \leq \eta_{6}$ and $\left|Q^{1}\right| \leq \eta_{6}$, and continue this way to the $(-2)$-degree position. Then turn over again by choosing any element in the kernel of $\operatorname{Hom}\left(E, P^{1}\right) \rightarrow \operatorname{Hom}\left(E, P^{0}\right)$ and go forward to the 2-degree position, then turn back again etc.

The direct union of this system of complexes will provide a new $\operatorname{Hom}(E,-)$ exact complex of injective objects, $C^{*}$, that might be not exact. But from $C^{*}$ we start a new zig-zag as the one which gave us the complex $H^{*}$, to get another exact complex of injectives. From this last one we get an $\operatorname{Hom}(E,-)$-exact complex of injectives, etc.

So we see that the direct limit of this process is a complex

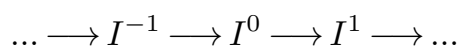

of injective objects that is exact, $\operatorname{Hom}\left(\mathcal{I}_{n j},-\right)$-exact, $\left|I^{i}\right|<\vartheta$ for some cardinal number $\vartheta$ that is independent of the choice of any of the objects in the process, and $\operatorname{ker}\left(I^{0} \rightarrow I^{1}\right)$ contains $S$.

Theorem 5.4. If the class of all Gorenstein injective objects of a locally noethe rian Grothendieck category is closed under direct products, then it is preenveloping.

Of course, if direct products are exact then the class of Gorenstein injective objects is closed under direct products, but the converse is not true as Krause showed in [21, Example 4.9]: he proved that if $K$ is a field, then direct products are not exact in the category $Q \operatorname{coh} \mathbb{P}_{K}^{1}$ of quasi-coherent sheaves over $\mathbb{P}_{K}^{1}$. However, direct products of Gorenstein injective objects in $Q \operatorname{coh} \mathbb{P}_{K}^{1}$ are Gorenstein injective since $Q \operatorname{coh} \mathbb{P}_{K}^{1}$ is locally noetherian and $\mathbf{D}\left(Q \operatorname{coh} \mathbb{P}_{K}^{1}\right)$ is compactly generated, so [21, Corollary 4.4] applies. This gives a great importance to a natural question about Gorenstein injective objects whose answer is still unknown: when are direct products of Gorenstein injective objects Gorenstein injective? In this paper we will give a couple of results in this direction whose ideas were obtained from J.E. Roos ([24, Theorem 1.3]). From now on we denote by $\mathcal{G I}$ the class of all Gorenstein injective objects.

Concerning these two questions (when is $\mathcal{G} \mathcal{I}$ closed under direct products? and, when do Gorenstein injective preenvelopes always exist?) in locally noetherian Grothendieck categories, two conditions can be found in the literature:

i) Krause: the derived category is compactly generated (see for instance [21, Corollary 4.4]).

ii) Hovey and Gillespie: the category has a system of generators of finite projective dimension (see for instance [16, Corollary 7.7]). 
In this paper we will add one more approach to the question by establishing a new condition to be satisfied by the category:

iii) it has a generator lying in the class ${ }^{\perp} \mathcal{G} \mathcal{I}$.

There is a question that immediately comes to mind: are these three conditions different or they turn out to be equivalent? There is no answer to this question so far (or we don't know it if there is one) so here is a problem to be investigated. However there are some partial answers that we can immediately give: $i i) \Rightarrow$ iii) is always true as one can see in [16, Lemma 7.2]. And if the generators of ii) are finitely generated then $i i) \Rightarrow i$ ) also holds by [16, Corollary 5.10].

Proposition 5.5. If the locally noetherian Grothendieck category has a generator in the class ${ }^{\perp} \mathcal{G} \mathcal{I}$ then any direct product of exact and $\operatorname{Hom}(\mathcal{I} n j,-)$-exact complexes with injective components is again exact. Thus, $\mathcal{G I}$ is closed under direct products.

Proof. Let $H \in^{\perp} \mathcal{G} \mathcal{I}$ be a generator of the category $\mathcal{A}$. By Gabriel-Popescu Theorem we know that $\operatorname{Hom}_{\mathcal{A}}(H,-)$ has an exact left adjoint functor $F$. Thus, $\operatorname{Hom}_{\mathcal{A}}(H,-)$ preserve injective objects.

Now, let $\left\{E_{i} ; i \in I\right\}$ be any family of exact and $\operatorname{Hom}(E,-)$-exact complexes with injective components, and call $G_{i}$ its 0-syzygy (so every $G_{i}$ is Gorenstein injective).

The product of the $E_{i}^{\prime} s$ is computed as $\prod^{\mathcal{A}} E_{i}=F\left(\prod \operatorname{Hom}_{\mathcal{A}}\left(H, E_{i}\right)\right)$, where $\prod \operatorname{Hom}_{\mathcal{A}}\left(H, E_{i}\right)$ is computed in the category $\operatorname{Mod}-\operatorname{End}(H)$ and so $\prod$ is exact.

But $F$ is also exact and $\operatorname{Hom}_{\mathcal{A}}\left(H, E_{i}\right)$ are all exact complexes since $H \in{ }^{\perp} \mathcal{G} \mathcal{I}$, so we see that $\prod^{\mathcal{A}} E_{i}$ is an exact complex.

$\prod^{\mathcal{A}} E_{i}$ being exact implies that its zero syzygy is $\prod G_{i}$, and $\prod^{\mathcal{A}} E_{i}$ is clearly $\operatorname{Hom}_{\mathcal{A}}(\mathcal{I} n j,-)$-exact, so we get that $\prod G_{i}$ is indeed Gorenstein injective since, of course, every component of $\prod^{\mathcal{A}} E_{i}$ is injective.

If we call $\prod^{(i)}$ the $i^{t h}$-derived functor of $\prod$, given any family of exact sequences $0 \rightarrow A_{i} \rightarrow B_{i} \rightarrow C_{i} \rightarrow 0$, we get the long exact sequence:

$0 \rightarrow \prod A_{i} \rightarrow \prod B_{i} \rightarrow \prod C_{i} \rightarrow \prod^{(1)} A_{i} \rightarrow \prod^{(1)} B_{i} \rightarrow \prod^{(1)} C_{i} \rightarrow \prod^{(2)} A_{i} \rightarrow \ldots$

Proposition 5.6. Let $\mathcal{A}$ be any Grothendieck category (either locally finitely presented or not). The following assertions are equivalent.

1. $\prod^{(1)} G_{i}=0$ for every family of Gorenstein injective objects $\left\{G_{i} ; i \in I\right\}$. That is, direct products are exact in the class of all Gorenstein injective objects.

2. $\prod^{(k)} G_{i}=0$ for every family of Gorenstein injective objects $\left\{G_{i} ; i \in I\right\}$ and every $k \geq 1$.

3. Any direct product of exact and $\operatorname{Hom}(\mathcal{I} n j,-)$-exact complexes with injective components is again exact and $\operatorname{Hom}(\mathcal{I} n j,-)$-exact. 
Proof. $1 . \Rightarrow 2$. Clear from the associated long exact sequence.

2. $\Rightarrow 3$. Let $\left\{E_{i} ; i \in I\right\}$ be any family of $\operatorname{Hom}(\mathcal{I} n j,-)$-exact exact complexes with injective components, and let $K_{i}^{n}=\operatorname{ker}\left(E_{i}^{n} \rightarrow E_{i}^{n+1}\right)$ for any $i$. Then for any $i$ and any $n$ we have an exact sequence

$$
0 \longrightarrow K_{i}^{n} \longrightarrow E_{i}^{n} \longrightarrow K_{i}^{n+1} \longrightarrow 0
$$

Since $K_{i}^{n}$ and $K_{i}^{n+1}$ are all Gorenstein injective objects, the sequence

$$
0 \longrightarrow \prod_{i} K_{i}^{n} \longrightarrow \prod_{i} E_{i}^{n} \longrightarrow \prod_{i} K_{i}^{n+1} \longrightarrow 0
$$

is exact by hypothesis. But then $\prod_{i} K_{i}^{n}$ is the kernel of $\prod_{i} E_{i}^{n} \rightarrow \prod_{i} E_{i}^{n+1}$ and also the cokernel of $\prod_{i} E_{i}^{n-1} \rightarrow \prod_{i} E_{i}^{n}$ for every $i$ and every $n$. This means that $\prod_{i} E_{i}$ is exact and so it is also $\operatorname{Hom}(\mathcal{I} n j,-)$-exact.

$3 . \Rightarrow 1$. Now, given any set $I$, let $G_{i}$ be a Gorenstein injective object for any $i \in I$ coming from the complex $E_{i}$.

By hypothesis we know that $\prod_{i} E_{i}$ is an exact and $\operatorname{Hom}(\mathcal{I} n j,-)$-exact complex of injectives, and since kernels and products are limits we have $Z^{0}\left(\prod_{i} E_{i}\right)=$ $\prod_{i} Z^{0}\left(E_{i}\right)=\prod_{i} G_{i}$

\section{Special Gorenstein injective preenvelopes}

In this section we want to give one more step in the study of Gorenstein injective preenvelopes in Grothendieck categories: we want to know when we can guarantee the existence of special Gorenstein injective preenvelopes.

In [5, Theorem 2.5] it is proved that if a class $\mathcal{F}$ of objects in a Grothendieck category is closed under direct sums, extensions and well ordered direct limits, then, if the cotorsion theory $\left(\mathcal{F}, \mathcal{F}^{\perp}\right)$ is cogenerated by a set, then any object $M$ in the category has a special $\mathcal{F}^{\perp}$-preenvelope. Following the proof one easily sees that it is enough to assume that the class $\mathcal{F}$ is closed under continuous direct unions. We will make use of this in the next result.

Recall that small cotorsion theories $(\mathcal{F}, \mathcal{G})$ in a Grothendieck category were defined in [20] as those cotorsion theories cogenerated by a set $\mathcal{S}$ and verifying: $\mathcal{F}$ contains a generator of the category and, for each $S \in \mathcal{S}$ there is a monomorphism $i_{S}$ with cokernel $S$ such that if $\operatorname{Hom}_{\mathcal{A}}\left(i_{S}, X\right)$ is surjective for all $S \in \mathcal{S}$, then $X \in \mathcal{G}$. Later, Estrada et al. proved in [12, Lemma 4.4] that the second condition above was redundant, so finally the cotorsion theory $(\mathcal{F}, \mathcal{G})$ is small provided that it is cogenerated by a set and that the class $\mathcal{F}$ contains a generator of the category. 
Theorem 6.1. Let $\mathcal{A}$ be locally noetherian and call $\mathcal{E}$ the class of all exact and $\operatorname{Hom}(\mathcal{I} n j,-)$-exact complexes with injective components and with cycles in $H^{\perp}(H$ is a generator of $\mathcal{A})$. Then $\mathcal{E}$ is special preenveloping in $C(\mathcal{A})$ and $\mathcal{Z}(\mathcal{E})$ is special preenveloping in $\mathcal{A}$. In particular, if $H$ is in ${ }^{\perp} \mathcal{G I}$ then the class of all exact and $\operatorname{Hom}(\mathcal{I} n j,-)$-exact complexes of injectives is special preenveloping in $C(\mathcal{A})$ and the class $\mathcal{G} \mathcal{I}$ is special preenveloping in $\mathcal{A}$.

Proof. The particular case follows easily from the first assertion, and if we let $\mathcal{Y}=\mathcal{I} n j$ and $\mathcal{T}=(\{H\} \cup \mathcal{Y})^{\perp}$ we see that $\mathcal{E}$ is the class of all exact complexes with components in $\mathcal{Y}$ and cycles in $\mathcal{T}$. In addition to that, it is clear that $\mathcal{E}[1]=\mathcal{E}$ and that $T \subseteq \mathcal{Y}^{\perp}$, so Proposition 3.1 applies, that is, if we prove that $\mathcal{E}$ is special preenveloping in $C(\mathcal{A})$ then $\mathcal{Z}(\mathcal{E})$ will be special preenveloping in $\mathcal{A}$.

Now, since $\left\{D^{n}(H / V) ; V \leq H, n \in \mathbb{Z}\right\}$ is a system of generators of $C(\mathcal{A})$, we can use Baer's Criterion to see that $\left\{D^{n}(H / V) ; V \leq H, n \in \mathbb{Z}\right\}^{\perp}=C(\mathcal{I} n j)$ (the class of all complexes of injectives), and using similar ideas to those of [1, Theorem 4.1] it is not hard to check that, if we let $E$ be the direct sum of one isomorphic copy of each indecomposable injective object, indeed

$$
\left(\left\{D^{n}(H / V) ; n \in \mathbb{Z}, V \leq H\right\} \cup\left\{S^{n}(E) ; n \in \mathbb{Z}\right\} \cup\left\{S^{n}(H) ; n \in \mathbb{Z}\right\}\right)^{\perp}=\mathcal{E} .
$$

Therefore $\left({ }^{\perp} \mathcal{E}, \mathcal{E}\right)$ is a small cotorsion theory cogenerated by a set, so by $[15$, Lemma 3.6], ${ }^{\mathcal{E}}$ is closed under continuous direct unions and then by $[5$, Theorem $2.5] \mathcal{E}$ is special preenveloping.

If a class $\mathcal{F}$ of a category $\mathcal{A}$ is closed under direct summands and every object of $\mathcal{A}$ has a special $\mathcal{F}$-preenvelope, then the pair $\left({ }^{\perp} \mathcal{F}, \mathcal{F}\right)$ is indeed a cotorsion theory, for if $A$ is any object of $\left({ }^{\perp} \mathcal{F}\right)^{\perp}$ we can consider a special $\mathcal{F}$-preenvelope

$$
0 \longrightarrow A \longrightarrow F \longrightarrow F^{\prime} \longrightarrow 0 \text {. }
$$

Now $F^{\prime} \in{ }^{\perp} \mathcal{F}$ means that $\operatorname{Ext}\left(F^{\prime}, A\right)=0$ and so the sequence splits, which means by hypothesis that $A \in \mathcal{F}$. Thus, in particular we deduce from Theorem 6.1 that when the class $\mathcal{G I}$ is closed under direct summands, the pair $\left({ }^{\perp} \mathcal{G I}, \mathcal{G I}\right)$ is a cotorsion theory (in any locally noetherian Grothendieck category with a generator lying in ${ }^{\perp} \mathcal{G I}$ ). But we can still say more about this cotorsion theory: we have already mentioned that Enochs et al. proved that when the category is Gorenstein, then $(\mathcal{L}, \mathcal{G I})$ is a complete hereditary cotorsion theory (where $\mathcal{L}$ is the class of all objects of finite injective dimension), and the same did Yang and Ding in a locally noetherian Grothendieck category with a generator of finite injective dimension. Though we don't know how to describe the class ${ }^{\perp} \mathcal{G} \mathcal{I}$ when the generator is not of finite injective dimension (for instance, if a noetherian ring $R$ is not of finite injective dimension then it does not hold in $\mathcal{L}$ but it always lies in ${ }^{\perp} \mathcal{G} \mathcal{I}$ ), we can still prove that 
the pair $\left({ }^{\perp} \mathcal{G} \mathcal{I}, \mathcal{G I}\right)$ is a complete hereditary cotorsion theory when ${ }^{\perp} \mathcal{G I}$ contains a generator of the category, which in part generalizes Yang and Ding's result. We will do so in the next result, where, in addition, we put together all the information we have about (special) $\mathcal{G} \mathcal{I}$-preenvelopes. We think this is useful in order to know where we are in the way of solving the problem we mentioned before: when are direct products of Gorenstein injectives Gorenstein injective?

Proposition 6.2. Let $\mathcal{A}$ be a locally noetherian Grothendieck category and consider the following statements:

1. $\mathcal{A}$ has a generator $H$ lying in ${ }^{\perp} \mathcal{G I}$.

2. $\left({ }^{\perp} \mathcal{G I}, \mathcal{G I}\right)$ is a complete hereditary cotorsion theory.

3. $\mathcal{G I}$ is special preenveloping.

4. $\mathcal{G I}$ is preenveloping.

5. $\mathcal{G I}$ is closed under direct products.

Then the following implications hold:

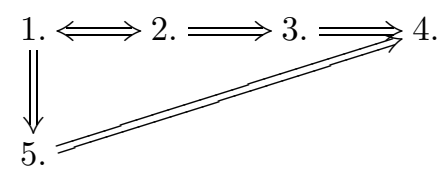

If $\mathcal{G I}$ is closed under direct summands then $4 . \Rightarrow 5$. holds too.

Proof. $1 . \Rightarrow 2$. We first have to see that $\left({ }^{\perp} \mathcal{G} \mathcal{I}, \mathcal{G I}\right)$ is a cotorsion theory, for which we just need to prove, by the comments above, that $\mathcal{G I}$ is closed under direct summands.

The dual arguments to those given in [19, Theorem 2.5] prove that $\mathcal{G I}$ is closed under cokernels of monomorphisms in any Grothendieck category, so by Proposition 5.5 we can use Eilenberg's swindle to see that indeed $\mathcal{G} \mathcal{I}$ is closed under direct summands. Therefore $\left({ }^{\perp} \mathcal{G} \mathcal{I}, \mathcal{G I}\right)$ is a cotorsion theory.

To prove that $\left({ }^{\perp} \mathcal{G I}, \mathcal{G I}\right)$ is complete we only need to check, by Theorem 6.1 , that for every object $X$ of $\mathcal{A}$ there is an exact sequence

$$
0 \longrightarrow G \longrightarrow G^{\prime} \longrightarrow X \longrightarrow 0
$$

with $G \in \mathcal{G I}$ and $G^{\prime} \in{ }^{\perp} \mathcal{G I}$. But we know there are two exact sequences $0 \rightarrow K \rightarrow$ $H^{(I)} \rightarrow X \rightarrow 0$ and $0 \rightarrow K \rightarrow G \rightarrow P \rightarrow 0$ with $G \in \mathcal{G I}$ and $P \in{ }^{\perp} \mathcal{G I}$ (given by Theorem 
6.1), so we get a pushout diagram

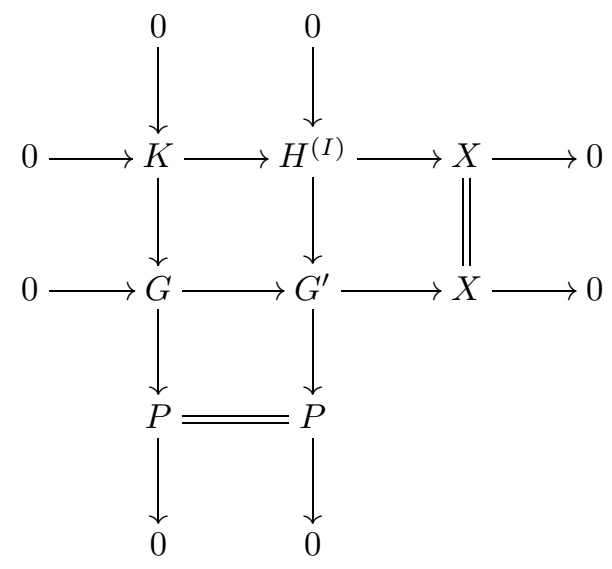

Now, $H \in{ }^{\perp} \mathcal{G I} \Rightarrow H^{(I)} \in{ }^{\perp} \mathcal{G} \mathcal{I}$, and since $P \in{ }^{\perp} \mathcal{G I}$ we get that $G^{\prime} \in{ }^{\perp} \mathcal{G I}$.

It only remains to prove that $\left({ }^{\perp} \mathcal{G I}, \mathcal{G I}\right)$ is hereditary, and since $\mathcal{A}$ has enough injectives, this will be done by proving that $\mathcal{G I}$ is closed under cokernels of monomorphisms. But this is just the categorical version of [9, Theorem 10.1.4].

2. $\Rightarrow 1$. Let $G$ be any generator of $\mathcal{A}$. By hypothesis there is an object $A \in{ }^{\perp} \mathcal{G I}$ and an epimorphism $A \rightarrow G$, which means that $A$ is also a generator of $\mathcal{A}$.

$2 . \Rightarrow 3$. and $3 . \Rightarrow 4$. Nothing to prove.

$5 . \Rightarrow 4$. Theorem 5.4.

1. $\Rightarrow 5$. Proposition 5.5.

Now, if $\mathcal{G I}$ is closed under direct summands then $4 . \Rightarrow 5$. is given by Theorem 5.2 .

Remark We have recently learnt that, under the assumption that $\mathcal{A}$ has a system of generators of finite projective dimension, Gillespie proved in [16, Corollary 7.7] the second part of the particular case of our Theorem 6.1 (the class $\mathcal{G} \mathcal{I}$ is special preenveloping in $\mathcal{A}$ ). We thank the referees for pointing out such a similarity.

\section{Applications}

There are many interesting categories where the problem of the existence of Gorenstein injective preenvelopes and Gorenstein flat precovers remains open. We now give a list of some classical Grothendieck categories of special interest in which 
the results proved in this paper apply and so the existence of such preenvelopes or precovers is guaranteed.

Examples 1. The category $G$-DMod of all discrete $\mathbb{Z}[G]$-modules over a profinite group $G$ is locally noetherian (see for instance [7]).

2. If $R$ is any ring with identity and $Q$ is any quiver, the category of representations of $Q$ by $R$-modules, $(Q, R$-Mod), is locally finitely presented and has a projective generator which of course lies in ${ }^{\perp} \mathcal{G} \mathcal{I}$. But $(Q, R$-Mod) having a projective generator means that the class of flat representations (with respect to the usual tensor product $-\otimes_{R Q} F$ ) of $Q$ is closed under extensions. Furthermore it is locally noetherian when the ring and the quiver are noetherian (see [6, Proposition 3.2]).

3. If $X$ is a quasi-compact and separated scheme, the category $Q \operatorname{coh}(X)$ of its quasi-coherent sheaves is locally finitely presented (see [17, I.6.9.12]), and it is locally noetherian whenever $X$ is a noetherian scheme (see [18, pg. 121]). Flat quasicoherent sheaves are closed under extensions since the categories of quasi-coherent sheaves and that of quasi-coherent modules over a certain quiver $Q$ are equivalent (see [3, Section 2]). In addition to that, it is well known that the derived category $\mathcal{D}(Q \operatorname{coh}(X))$ is compactly generated (see for instance [23, Proposition 2.5]), so by $[21$, Lemma 4.4] we get that the class $\mathcal{G} \mathcal{I}$ is closed under direct products.

4. If $R=\oplus_{g \in G} R_{g}$ is a $G$-graded ring ( $G$ is a group), then the category $R$-gr of $G$-graded $R$-modules is locally finitely presented with a projective generator (so it holds in ${ }^{\perp} \mathcal{G I}$ and the class of flat graded modules is closed under extensions), and it is locally noetherian whenever $R$ is a graded noetherian ring (see [22]).

Therefore the class $\mathcal{G F}$ of all Gorenstein flat objects (with respect to any tensor product which makes the class of flat objects to be closed under extensions) of any of the above categories is precovering. Thus, in particular, the class $\mathcal{G} \mathcal{F}$ with respect to the usual tensor product in each one of the categories $(Q, R$-Mod), $Q \operatorname{coh}(X)$ and $R$-gr is precovering. The usual tensor product in the category $G$-DMod is

$$
A \otimes_{\mathbb{Z}[G]}-: G-\mathrm{DMod} \longrightarrow \mathcal{A} b
$$

( $A$ is a discrete module), and for the class $\mathcal{G F}$ (with respect to this tensor product) in this category to be precovering, we need the class of flat discrete modules to be closed under extensions, which we don't know so far. On the other hand, when the categories $Q \operatorname{coh}(X),(Q, R$-Mod) or $R$-gr are locally noetherian then the class $\mathcal{G} \mathcal{I}$ is preenveloping in $Q \operatorname{coh}(X)$ and special preenveloping in $(Q, R)$-Mod and $R$-gr.

It is worth noting that recently, Christensen, Estrada and Iacob proved that the class of Gorenstein flat quasi-coherent sheaves over any scheme (Gorenstein flat with respect to the usual tensor product of modules) is precovering (see [2, Corollary 4.8]). Our type of precovers are somehow more general than the ones 
found by them, but our assumptions on the structure scheme are not needed for them.

We now give a last application concerning the categories of complexes on a Grothendieck category.

Proposition 7.1. If $\mathcal{A}$ is a Grothendieck category satisfying any of the following properties, then the same is true for $C(\mathcal{A})$ :

1. $\mathcal{A}$ is locally finitely presented.

2. $\mathcal{A}$ is locally noetherian and the class of exact complexes with injective components is closed under direct products.

3. $\mathcal{A}$ is locally noetherian and has a generator in ${ }^{\perp} \mathcal{G I}$.

Therefore, the class $\overline{\mathcal{G F}}$ of Gorenstein flat objects in $C(\mathcal{A})$ (with respect to any tensor product which makes the class of flat complexes to be closed under extensions) is precovering under condition 1., and the class $\overline{\mathcal{G I}}$ is preenveloping under condition 2. and special preenveloping under condition 3 .

Proof. If $\mathcal{A}$ is locally finitely presented (locally noetherian) and $\mathcal{X}$ is a set of finitely presented (noetherian) generators of $\mathcal{A}$, then $\left\{D^{n}(X) ; X \in \mathcal{X}, n \in \mathbb{Z}\right\}$ is a set of finitely presented (noetherian) generators of $C(\mathcal{A})$.

Now, direct products in $C(\mathcal{A})$ are computed componentwise so if the class of exact complexes on $\mathcal{A}$ with injective components is closed under direct products, then the same is true for the class of exact complexes on $C(\mathcal{A})$ with injective components.

Finally, since $\operatorname{Hom}_{\mathcal{A}}\left(E, X^{i}\right)=\operatorname{Hom}_{C(\mathcal{A})}\left(D^{i}(E), X\right)$ for any complex $X$ in $C(\mathcal{A})$ and any object $E$ of $\mathcal{A}$, we see that Gorenstein injective complexes have Gorenstein injective components, so if $\mathcal{A}$ has a generator $H$ in ${ }^{\perp} \mathcal{G} \mathcal{I}$ then any $D^{i}(H)$ holds in $\perp \overline{\mathcal{G I}}$ (where $\overline{\mathcal{G I}}$ is the class of Gorenstein injective complexes), and then $C(\mathcal{A})$ has a generator in $\perp \overline{\mathcal{G I}}$.

Acknowledgments. The authors wish to thank the work done by the two referees of the paper. Their comments helped us to clarify some aspects of the paper and to make a better version of it.

The authors J.R. García Rozas, L. Oyonarte, and B. Torrecillas were partially supported by the grant MTM2014-54439-P from MINECO (Spain).

\section{References}

1. Bravo, D., Gillespie, J. and Hovey, M., The stable module category of a general ring. arXiv: 1405 . 57 .68v1. 
2. Christensen, L. W., Estrada and Iacob A, S., A Zariski-local notion of F-total acyclicity for complexes of sheaves, Quaest. Math. 40 (2017), 197-215.

3. Enochs, E. and Estrada, S., Relative homological algebra in the category of quasicoherent sheaves, Adv. Math. 194 (2005), 284-295.

4. Enochs, E., Estrada, S. and García Rozas, J. R., Gorenstein categories and Tate cohomology on projective schemes, Math. Nachr. 281 (2008), 525-540.

5. Enochs, E., Estrada, S., García Rozas, J. R. and Oyonarte, L., Flat covers in the category of quasi-coherent sheaves over the projective line, Comm. Algebra 32 (2004), 1497-1508.

6. Enochs, E., García Rozas, J. R., Oyonarte, L. and Park, S., Noetherian quivers, Quaest. Math. 25 (2002), 531-538.

7. Enochs, E., García Rozas, J. R., Oyonarte, L. and Torrecillas, B., On Gorenstein injective discrete modules over profinite groups, Acta Math. Hungar. 142 (2014), 296-316.

8. Enochs, E. and KAhn, E., Flat discrete modules over profinite groups, Comm. Algebra 42 (2014), 2331-2337.

9. Enochs, E. and Jenda, O., Relative homological algebra, 2nd ed., De Gruyter Expositions in Mathematics 30, Berlin/Boston (2011).

10. Enochs, E., Oyonarte, L. and Torrecillas, B., Flat covers and flat representations of quivers, Comm. Algebra 32 (2004), 1319-1338.

11. Estrada, S. and Gillespie, J., The projective stable category of a coherent scheme, Proc. Roy. Soc. Edinburgh Sect. A 149 (2019), 15-43.

12. Estrada, S., Guil Asensio, P. A., Prest, M. and Trlifaj, J., Model category structures arising from Drinfeld vector bundles, Adv. Math. 231 (2012), 14171438.

13. García Rozas, J. R., Covers and envelopes in the category of complexes of modules, Research Notes in Mathematics 407, Chapman and Hall/CRC, London/New York/Washington, 1999.

14. Gillespie, J., Cotorsion pairs and degreewise homological model structures, Homology, Homotopy Appl. 10, N.1 (2008), 283-304.

15. Gillespie, J., Kaplansky classes and derived categories, Math. Z. 257 (2007), 811843.

16. Gillespie, J., Models for homotopy categories of injectives and Gorenstein injectives, Comm. Algebra 45 (2017), 2520-2545.

17. Grothendieck, A. and Dieudonné, J. A., Eléments de géométrie algèbrique I, Grundlehren math. Wiss. 166, Springer, Berlin-Heidelberg-New York, 1971.

18. Hartshorne, R., Residues and duality, LNM 20, Springer, Berlin, 1966.

19. Holm, H., Gorenstein homological dimensions, J. Pure Appl. Algebra 189 (2004), $167-193$.

20. Hovey, M., Cotorsion pairs, model category structures, and representation theory, Math. Z. 241 (2002), 553-592.

21. Krause, H., The stable derived category of a noetherian scheme, Compos. Math 141 (2005), 1128-1162.

22. NǍstǎsescu, C. and Van Oystaeyen, F., Methods of graded rings, LNM 1836, Springer, Berlin, 2004.

23. Neeman, A., The Grothendieck duality theorem via Bousfield's techniques and Brown 
representability, J. Amer. Math. Soc. 9 (1996), 205-236.

24. Roos, J.-E., Derived functors of inverse limits revisited, J. Lond. Math. Soc. (2) 73 (2006), 65-83.

25. SAORÍn, M. and ŠŤOvíČEK, J., On exact categories and applications to triangulated adjoints and model structures, Adv. Math. 228 (2011), 968-1007.

26. Stenström, B., Rings of quotients, Springer, Berlin, 1975.

27. ŠŤOvíčEK, J., Deconstructibility and the Hill Lemma in Grothendieck categories, Forum Math. 25 (2013), 193-219.

28. YAng, X. and Ding, N., On a question of Gillespie, Forum Math. 27 (2015), 32053231.

29. YAng, G. and LiAng, L., All modules have Gorenstein flat precovers, Comm. Algebra 42 (2014), 3078-3085.

Edgar Enochs

Dept. of Mathematics

University of Kentucky

Lexington

KY 40506-0027

U.S.A.

e.enochs@uky.edu

J.R. García Rozas

Dept. of Mathematics

University of Almería

04071

Almería

Spain

jrgrozas@ual.es
Luis Oyonarte

Dept. of Mathematics

University of Almería

04071

Almería

Spain

oyonarte@ual.es

Blas Torrecillas

Dept. of Mathematics

University of Almería

04071

Almería

Spain

btorreci@ual.es

Received January 17, 2017

in revised form March 2, 2018 